\title{
Comportamento Social, Produção Agregada e Prática Cultural: Uma Análise Comportamental de Fenômenos Sociais ${ }^{1}$
}

\author{
Angelo Augusto Silva Sampaio ${ }^{2}$ \\ Universidade Federal do Vale do São Francisco (UNIVASF) \\ Maria Amalia Pie Abib Andery ${ }^{3}$ \\ Pontifícia Universidade Católica de São Paulo (PUC-SP)
}

\begin{abstract}
RESUMO - A Análise do Comportamento deve e tem lidado com os fenômenos sociais: fatos ou eventos envolvendo as ações de mais de uma pessoa. A partir da literatura, buscou-se distinguir três eventos rotulados como fenômenos sociais: comportamento social - contingência tríplice cujas consequências são mediadas pelo comportamento operante de outro(s) indivíduo(s); produção agregada - conjunto de comportamentos de mais de um indivíduo que geram um produto agregado; e prática cultural - comportamentos aprendidos similares propagados por sucessivos indivíduos. Características e complexidades destes três fenômenos são discutidas. Possibilidades de diálogo com as Ciências Sociais são ressaltadas.
\end{abstract}

Palavras-chave: comportamento social; produção agregada; prática cultural; Análise do Comportamento.

\section{Social Behavior, Aggregated Production, and Cultural Practice: A Behavioral Analysis of Social Phenomena}

\begin{abstract}
Behavior Analysis ought to deal with and has effectively dealt with social phenomena: facts or events involving actions of more than one person. Based on the published literature, this paper seeks to distinguish three events labeled as social phenomena: social behavior - three-term contingencies whose consequences are mediated by the operant behavior of another organism(s); aggregated production - sets of behaviors of more than one individual that result in an aggregated product; and cultural practice - similar learned behaviors propagated by successive individuals. Characteristics and complexities of these three phenomena are discussed. Dialogue possibilities with Social Sciences are highlighted.
\end{abstract}

Keywords: social behavior; aggregated production; cultural practice; Behavior Analysis.

O objeto de estudo da Análise do Comportamento é a ação $0^{4}$ de organismos individuais. Seus princípios, conceitos e métodos já foram aplicados ao estudo das ações dos mais diversos seres vivos, mas o interesse principal desse campo de conhecimento recai sobre o comportamento humano (Andery, 1990; Skinner, 1953). Ora, o mundo dos seres humanos é formado em grande parte por outros seres humanos e mesmo o ambiente "físico" no qual vivem é, em boa medida, construído por sua própria espécie. Se a compreensão do comportamento humano, como o de outras espécies, depende da análise das interações entre sujeito e ambiente, e se o ambiente humano é em grande parte composto pelas ações

1 Trabalho derivado da Dissertação de Mestrado do primeiro autor, sob orientação da segunda autora, apresentada ao Programa de PósGraduação em Psicologia Experimental: Análise do Comportamento (PUC-SP) e realizada com o apoio do Conselho Nacional de Desenvolvimento Científico e Tecnológico (CNPq). Os autores agradecem a Alexandre Dittrich, Nilza Micheletto, Tereza Sério e Hélder Gusso pelos comentários e sugestões em versões anteriores do trabalho.

2 Endereço para correspondência: Av. da Integração, 870, apt. 303, Alto Cheiroso. Petrolina, PE. CEP 56303-450. Tel. (87) 3862-9320. Email: angsampaio@uol.com.br.

3 Bolsista $\mathrm{CNPq}$ - Produtividade em Pesquisa.

4 Os termos ação e comportamento serão utilizados como sinônimos, ambos referindo-se à interação entre respostas de um organismo e estímulos do ambiente. de outras pessoas, logo, grande parte do comportamento humano é determinado por outros homens e mulheres. Podemos afirmar, portanto, que o interesse especial da Análise do Comportamento pelo comportamento humano a obriga a tratar de fenômenos sociais. O termo "fenômeno" refere-se a fatos ou eventos de interesse científico; e o termo "social" adjetiva algo concernente à sociedade, à comunidade ou ao coletivo, à união de várias pessoas (Houaiss, Villar \& Franco, 2001). Podemos entender fenômenos sociais, portanto, como fatos ou eventos de interesse científico envolvendo os comportamentos de várias pessoas (ou de mais de uma pessoa). Trata-se das interações e dos resultados das interações de pessoas agindo em conjunto.

Muito cedo, Skinner (e.g., 1948/1978, 1953) incluiu o estudo de fenômenos sociais como parte da Análise do Comportamento. A investigação empírica de muitos desses fenômenos, inclusive, já vem sendo conduzida há bastante tempo (Guerin, 1994; Schmitt, 1998). Contudo, muitas questões ainda permanecem em aberto. Uma questão central envolve o agrupamento de fenômenos diversos sob o mesmo rótulo (Andery, Micheletto \& Sério, 2005). Uma distinção entre fenômenos com propriedades semelhantes usualmente envolve algum grau de arbitrariedade, mas pode facilitar a interlocução entre estudiosos e permitir avanços na pesquisa e na intervenção. Autores como Glenn (1986, 1988, 2003, 2004; ver também Malott \& Glenn, 2006) e Guerin (1992, 
1994, 2001), além do próprio Skinner (1953, 1957/1992, 1981), propuseram diferenciações entre fenômenos sociais a partir da perspectiva da Análise do Comportamento. Nosso objetivo é, a partir do trabalho desses autores, tentar refinar o que chamamos de fenômenos sociais, distinguindo três eventos que são comumente tratados sob o mesmo rótulo: comportamento social, produção agregada e prática cultural.

\section{Comportamento Social}

O termo comportamento social pode sugerir uma contraposição com o que seria "comportamento individual", implicando que o modo como as pessoas agem, pensam, falam, aprendem etc. e/ou os princípios explicativos empregados na sua compreensão são diferentes em situações sociais e não sociais. É nesse sentido que expressões como "aprendizagem social", "cognição social", "dinâmica de grupo" e "fato social" são algumas vezes utilizadas. Para a Análise do Comportamento, entretanto, os princípios e conceitos construídos (principalmente) a partir do estudo do "comportamento individual" seriam suficientes para lidar com o que é normalmente rotulado de "comportamento social". Esse último apresentaria características próprias (talvez singulares), mas características que ainda podem ser descritas a partir dos mesmos fundamentos filosóficos, teóricos, metodológicos e conceituais válidos para todo tipo de comportamento (Andery \& Sério, 2006).

Assim, o termo "comportamento social" só se justificaria para enfatizar certas particularidades de um tipo ou subcategoria de comportamento (como todo comportamento, necessariamente "individual"). Na Análise do Comportamento, de fato, o termo tem sido utilizado sempre que uma ação envolve a participação ou mediação de outra pessoa como ambiente relevante para a ação analisada. O que pode ser aceito como participação ou mediação, entretanto, ainda é objeto de discussão (Guerin, 1994). Adotaremos a proposta de Skinner (1953, 1957/1992), que toma comportamento social como um tipo de comportamento operante e enfatiza o modo como suas consequências (geradas por outro indivíduo) são produzidas por uma classe de respostas. Nossa definição, portanto, enfatizará contingências tríplices, mais especificamente, a relação resposta-consequência.

Ao tratar de comportamento social, Skinner (1953, 1957/1992) de início parece igualá-lo a contingências tríplices cujas consequências são produzidas com a mera participação de outro(s) indivíduo(s). Em tais contingências, a outra pessoa "participa meramente... como um objeto físico" (Skinner, 1957/1992, p. 224). Skinner cita os exemplos de um boxeador desferindo um golpe no seu adversário e de um médico realizando uma cirurgia. Em ambos os casos, as consequências relevantes (acertar o adversário e remover um apêndice inflamado) não seriam produzidas sem a participação de outra pessoa. No entanto, a outra pessoa funciona como um objeto físico qualquer, seu comportamento não é relevante para a produção das consequências. Nesses casos, a produção das consequências relevantes para o comportamento é direta e pode ser explicada apenas pelas ciências físicas, sem recorrer às ciências do comportamento.
Skinner (1953, 1957/1992) restringe o termo comportamento social, contudo, a contingências tríplices cujas consequências relevantes comportamentalmente são produzidas pelo comportamento operante de outro(s) indivíduo(s). Aqui, o outro indivíduo não funciona como um mero objeto físico, ele age como um organismo vivo; as consequências são produzidas apenas por meio de uma ação sua. Nesse caso, Skinner refere-se não mais à participação, mas à mediação de outro indivíduo. As consequências mediadas podem ser: (1) as próprias respostas operantes, (2) as consequências (reforços ou punições); e/ ou (3) resultados diretos dessas respostas e consequências do outro indivíduo. Alguns exemplos seriam: um professor ensinando música a um aprendiz, uma criança pirraçando o pai para ele lhe comprar algo e todo e qualquer comportamento verbal. Nesses casos, o aprendiz, o pai e o ouvinte agem como organismos vivos e, além disso, não respondem “mecanicamente”. A Figura 1 ilustra as relações envolvidas no comportamento social. No exemplo dessa figura, apenas o comportamento do indivíduo A é, por definição, necessariamente social.

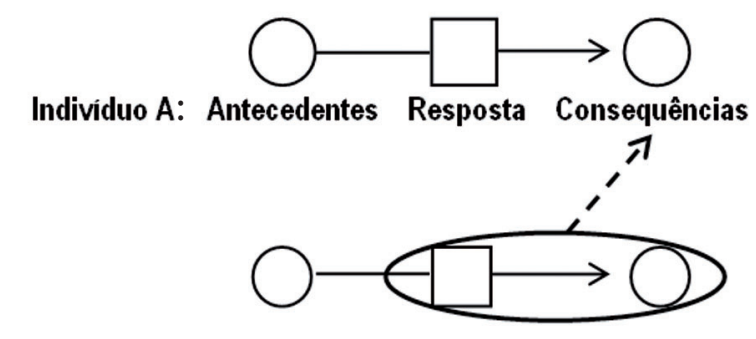

Individuo B: Antecedentes Resposta Consequências

Figura 1. Diagrama ilustrando a definição de comportamento social.

Comportamento social, assim, envolve o que Skinner (1953, pp. 201, 229, 310; 1957/1992, p. 432) chama de "sistemas entrelaçados de resposta" ou "sistemas entrelaçados de comportamento" (interlocking systems of response ou interlocking systems of behavior) ou "contingências entrelaçadas" (interlocking contingencies), expressões que enfatizam que contingências tríplices de dois indivíduos de certa forma se sobrepõem ou se cruzam: a resposta, ou um produto gerado pela resposta, ou a consequência em uma das contingências participa como consequência em outra contingência.

Consideramos comportamento social, portanto, qualquer contingência tríplice cujas consequências são mediadas pelo comportamento operante de outro(s) indivíduo(s). Essas contingências tríplices apresentam propriedades distintivas, que não são redutíveis a descrições das ciências físicas e só podem ser explicadas, portanto, pelas ciências do comportamento. Nesses casos, a produção das consequências exige a análise do comportamento de outro(s) indivíduo(s), ou seja, envolve - ainda que na sua origem - comportamento operante de outro(s) indivíduo(s) (Andery, Micheletto \& Sério, 2005). 


\section{Algumas questões quanto à definição de comportamento social}

A definição de comportamento social que adotamos levanta algumas questões. Uma dessas questões é se um indivíduo agindo sozinho pode se comportar socialmente. Guerin (2001), em posição semelhante a aquela defendida já em 1950 por Keller e Schoenfeld, responde afirmativamente a essa questão, enumerando 18 maneiras pelas quais agir sozinho poderia sim ser considerado comportamento social. $\mathrm{O}$ argumento central fundamenta-se na noção de que um operante é uma unidade estendida no tempo e que, como tal, sempre deve ser analisado considerando-se toda a história de interações entre suas classes de respostas e de estímulos, já que é essa história que determina suas propriedades. Assim, se as consequências de um operante já foram mediadas por outros indivíduos, independente de algumas de suas instâncias (respostas particulares) serem emitidas quando o indivíduo está sozinho, ele deveria sim ser considerado um comportamento social.

Outra questão que decorre da definição que adotamos de comportamento social é se indivíduos de outras espécies apresentam comportamento social. Nossa definição não implica limitações quanto à espécie dos indivíduos envolvidos em contingências tríplices sociais. Se as consequências do comportamento de um chimpanzé ao interagir com outro chimpanzé ou com um veterinário e de um veterinário ao interagir com outro veterinário ou com um chimpanzé forem mediadas pelo outro indivíduo emitindo comportamento operante, todos poderiam ser considerados comportamentos sociais.

Uma última questão relaciona-se com a ausência, em nossa definição, de limitações quanto ao modo pelo qual o comportamento operante de outro(s) indivíduo(s) afeta um comportamento social. Ele pode fazê-lo diretamente (e.g., quando um sorriso reforça um elogio), ou indiretamente (e.g., quando a visualização de uma mensagem de correio eletrônico - enviada por outro indivíduo - reforça o conectar-se à internet e ler emails). Os comportamentos que envolvem interações com objetos construídos por outros indivíduos (e.g., computadores) poderiam, então, ser considerados comportamento social? Aqui, o fundamental na identificação de um operante como social seria a identificação da mediação das consequências por outros indivíduos e também que o operante caracterize-se pelas propriedades distintivas do comportamento socialmente mediado, discutidas a seguir. Tais propriedades exigem que se empregue as ciências do comportamento para que se compreenda como se dá a mediação - direta ou indireta - das consequências no comportamento social. Tal necessidade justificaria um tratamento distinto do comportamento como comportamento social. Dessa análise, portanto, é que decorreria a classificação de um comportamento operante indiretamente mediado como social ou não.

\section{Propriedades distintivas do comportamento social}

Quais propriedades, portanto, justificam um termo e um tratamento distintos para lidar com relações comporta- mentais sociais? Com base nos trabalhos de Skinner (1953, 1957/1992), que apresentou diversas delas, e de Guerin (1992, 1994), que retomou os trabalhos de Skinner e também as especificou, podemos enumerar algumas das propriedades do comportamento social:

1) Suas consequências geralmente são condicionadas;

2) Suas consequências geralmente são condicionadas generalizadas;

a) Como muitas dessas consequências generalizadas são produzidas diretamente por outros indivíduos (e.g., "atenção", "sinais de aprovação" ou de "afeição"), aspectos da pessoa que gera tais consequências (e.g., sua mera presença) podem tornar-se os únicos antecedentes controlando a classe de respostas consequenciada, mesmo que tais aspectos não se relacionem com o critério de consequenciação vigente. Esse processo pode explicar, por exemplo, porque apenas olhar nos olhos de alguém pode produzir grandes alterações em seu comportamento;

b) Em parte devido a tais características, geralmente as consequências comportamentalmente relevantes não são óbvias ou facilmente mensuráveis; eventos, objetos ou características de objetos extremamente sutis podem ser consequências funcionais;

3) Como muitas vezes o outro indivíduo precisa de tempo para reagir, as consequências podem ser atrasadas em relação à resposta que as gerou;

4) Em geral, não há relação direta entre a energia da resposta e a magnitude das consequências produzidas (um sussurro, por exemplo, pode produzir uma gargalhada estrondosa do ouvinte);

5) O critério de consequenciação geralmente "varia de momento a momento, dependendo da condição do agente reforçador [isto é, do outro indivíduo]. Desta forma, respostas diferentes podem produzir o mesmo efeito, e uma [mesma] resposta pode produzir diferentes efeitos, dependendo da ocasião." (Skinner, 1953, p. 299) Ou seja, os esquemas de reforço e punição nos quais as consequências são arranjadas em geral são:

a) Intermitentes - o indivíduo gerador das consequências comumente não responde de modo tão "confiável" quanto o ambiente físico. Além do mais, diferentes esquemas intermitentes geram diferentes padrões de comportamento, explicando em parte a variabilidade das ações mantidas por tais esquemas;

b) Variáveis - esses esquemas tendem a gerar comportamento mais resistente à extinção e com frequência mais estável que esquemas fixos. Além disso, o comportamento assim mantido tende a ser mais extenso e flexível, já que mais variação é produzida quando a consequência não ocorre;

c) Ajustáveis - esses esquemas ajustam-se aos atos sendo consequenciados, por exemplo, como resultado do aumento da tolerância a controle 
aversivo ou do aumento de exigência para reforço por parte do outro indivíduo. As consequências raramente são independentes do comportamento reforçado, de modo que o outro indivíduo quase sempre fica sob controle das respostas consequenciadas;

d) Concorrentes - consequências diferentes tendem a estar disponíveis ao mesmo tempo para diferentes classes de respostas;

e) Complexos, combinados - os esquemas simples (intervalo variável - VI, intervalo fixo - FI, razão variável - VR, razão fixa - FR) são raros em situações sociais. Esquemas complexos (e.g., concorrentes encadeados com retenção temporária da disponibilidade do reforço) são a norma, produzindo repertórios comportamentais extensos e flexíveis, nos quais uma rápida alternação de uma classe de respostas para outra seria comum;

6) Os antecedentes, por serem determinados por uma história individual constituída de consequências mediadas por outras pessoas e arranjadas em esquemas complexos, também se diferenciam. De modo geral, eles são extremamente sutis, complexos e difíceis de identificar.

Essas propriedades são dificilmente encontradas em "comportamentos não-sociais" e são, portanto, típicas do comportamento social. Assim, uma compreensão adequada desse fenômeno deveria basear-se fortemente nos resultados de áreas de pesquisa tais como: consequências condicionadas, generalizadas e atrasadas; relações entre energia da resposta e magnitude das consequências; esquemas complexos de reforço; e o controle de estímulos estabelecido por todas essas contingências.

Como Skinner (1953) sugeriu, o comportamento social pode ser estudado como um fenômeno no âmbito do indivíduo, com propriedades distintivas, tomando-se a contingência tríplice como unidade de análise. Apesar de algumas características particulares e de sutilezas da sua análise, nenhum princípio ou conceito novo seria necessário para lidar com esse fenômeno. Os fenômenos sociais discutidos a seguir, contudo, embora possam ser compostos por contingências tríplices cujas consequências são mediadas por outros indivíduos, levantam a questão da necessidade de outros conceitos ou unidades de análise para que sejam adequadamente compreendidos e descritos.

\section{Produção Agregada}

Muitos problemas sociais são definidos não pelo que os indivíduos fazem, mas pelo resultado gerado por suas ações. Essas ações podem ser realizadas conjuntamente ou não; podem envolver topografias de comportamento semelhantes ou não; podem ser controladas pelas mesmas consequências ou não. O efeito estufa, por exemplo, é uma questão ambiental premente que resulta de muitas pessoas agindo de diferentes modos (utilizando automóveis, queimando florestas, traba- lhando em fábricas poluidoras etc.), em conjunto ou não, envolvendo diferentes topografias e sendo controladas por diferentes consequências. Fenômenos sociais desse tipo são delimitados não pelas relações funcionais entre respostas de um indivíduo e eventos ambientais, mas pelos resultados gerados pelas respostas dos indivíduos. As respostas que contribuem para tais resultados são emitidas por diversos indivíduos e não precisam ser controladas pelo próprio resultado ou pelas mesmas contingências. A inclusão de uma resposta como parte do fenômeno de interesse decorre de sua contribuição para um produto e não das relações que a controlam.

O conceito de produto agregado (aggregate outcome ou aggregate product) (Glenn, 1988, 1991; Glenn \& Malott, 2004; Malott \& Glenn, 2006) foi proposto para lidar com tais fenômenos sociais. Produtos agregados, tal como consequências comportamentais, são eventos subsequentes às respostas e por elas produzidos. Eles sempre são, no entanto, um resultado gerado pelas respostas de mais de uma pessoa. Uma pessoa sozinha, por exemplo, não é capaz de mover uma pedra de uma tonelada, mas 10 pessoas trabalhando juntas podem gerar o produto agregado "pedra deslocada".

Da mesma forma que uma consequência produzida pela resposta de um indivíduo pode não se constituir um evento ambiental para tal resposta, um produto agregado não precisa afetar as relações comportamentais que o produziram para ser rotulado enquanto tal. No exemplo do deslocamento da pedra de uma tonelada, o conjunto de pegadas criado pelas 10 pessoas também poderia ser considerado um produto agregado de seus comportamentos, apesar de provavelmente não afetar comportamentalmente essas pessoas. Ou seja, em algumas situações há retroação do produto agregado sobre os comportamentos que o produziram, enquanto, em outras, isso não ocorre.

$\mathrm{Na}$ discussão do comportamento social, nosso foco é o comportamento, isto é, as relações indivíduo-ambiente (outro indivíduo) constitutivas do fenômeno e, portanto, a ação individual é a variável a ser explicada. Quando nosso interesse migra para fenômenos sociais envolvendo produtos agregados, nosso foco muda do comportamento para as alterações ambientais produzidas pelo comportamento. Os comportamentos (as interações produtoras) envolvidos em tais fenômenos deixam de ser, em um primeiro momento, pelo menos, a variável a ser explicada e passam a ser variável que explica tais produtos.

Um fenômeno social envolvendo produto agregado pode ser chamado abreviadamente de produção agregada. Produções agregadas como são aqui definidas sempre envolvem ações de mais de um indivíduo. E tais ações não precisam ser necessariamente sociais - embora seja quase impossível falar em comportamento humano que não tenha "componentes" de comportamento social.

Há vários tipos de produções agregadas, de diferentes níveis de complexidade, englobando, por exemplo, desde duas pessoas juntas puxando uma corda, até a produção de um automóvel em uma grande empresa. Algumas características desses fenômenos permitem uma tentativa de classificação.

Em primeiro lugar, como já mencionamos, produtos agregados podem afetar (de diferentes modos) as relações comportamentais que os produziram ou não ter efeito algum sobre elas (Malott \& Glenn, 2006). 


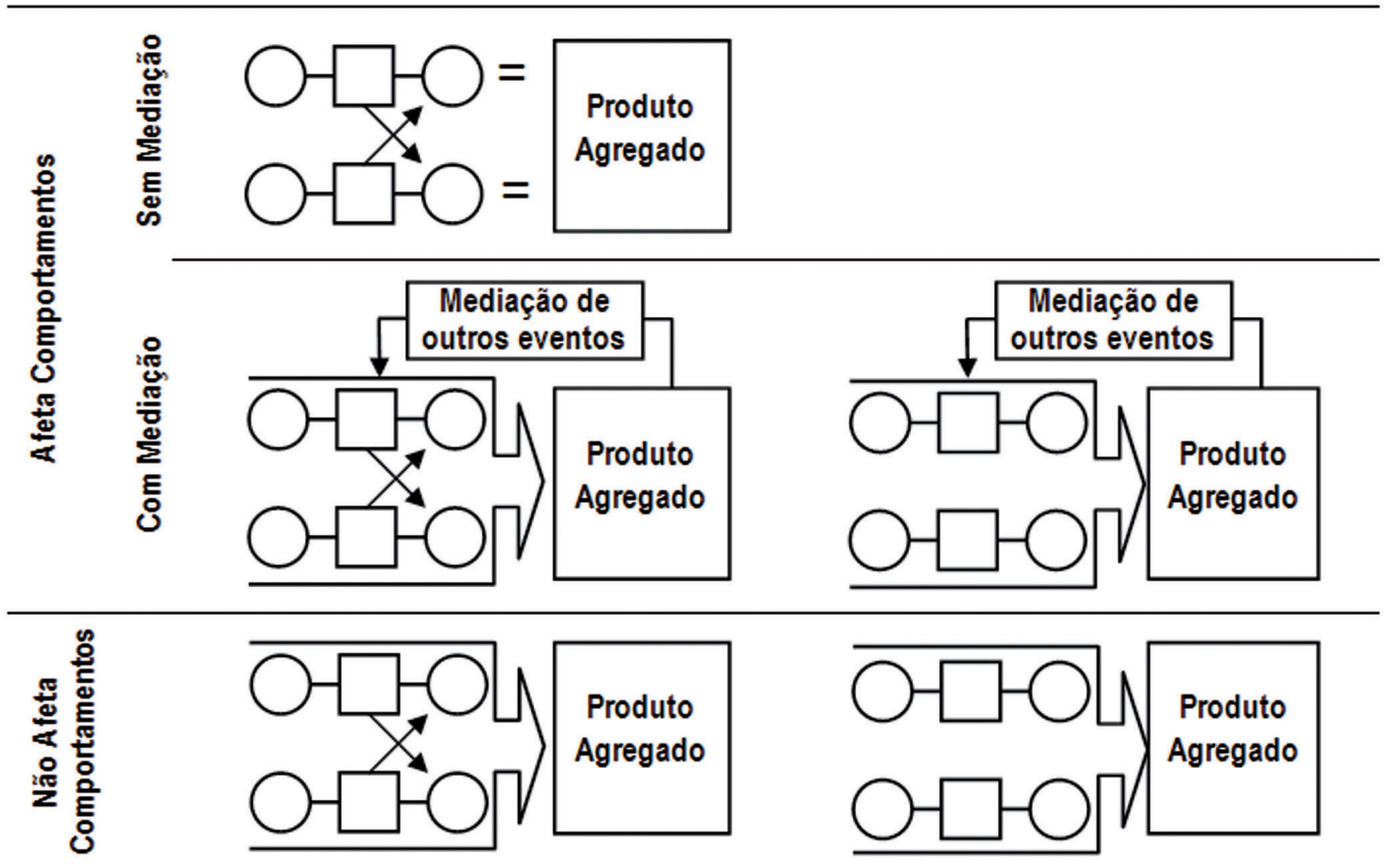

Figura 2. Representação esquemática de cinco tipos de produções agregadas. Cada conjunto de círculo-quadrado-círculo representa uma contingência tríplice (antecedentes-resposta-consequências) de um indivíduo específico; as setas pequenas indicam que a resposta de um indivíduo gera estímulos que afetam o comportamento de outro indivíduo, isto é, que as contingências são entrelaçadas.

Em segundo lugar, quando um produto agregado afeta os comportamentos que o produziram, ele pode fazê-lo com ou sem mediação de outros comportamentos. Os trabalhadores e administradores de uma fábrica que despeja resíduos tóxicos em um rio podem ser afetados por este produto agregado pela mediação de avisos das autoridades responsáveis pela proteção ambiental da área. Os comportamentos dos membros de um grupo também podem ser afetados por um produto agregado pela mediação de um de seus membros, quando, por exemplo, esse indivíduo descreve verbalmente as relações existentes entre comportamentos dos membros do grupo e o produto agregado (um engenheiro ambiental da fábrica poderia notar os prejuízos causados ao ambiente e propor alterações na produção). Por sua vez, quando um produto agregado afeta os comportamentos que o produziram sem nenhuma mediação (de outras pessoas ou de outros comportamentos), o produto coincide com consequências comportamentais. A produção de artesanato por um pequeno grupo pode ser mantida diretamente pelas peças produzidas (produtos agregados que coincidem com consequências comportamentais). Essa distinção é relevante porque as características da retroação do produto agregado sobre os comportamentos que o produziram possivelmente são diferentes quando há e quando não há mediação.
Em terceiro lugar, outra característica que distingue produções agregadas é o entrelaçamento (ou não) das contingências que geram o produto agregado . $^{5}$

Assim, propomos uma classificação das produções agregadas a partir de três distinções (sugeridas em parte por Malott \& Glenn, 2006): produtos agregados que afetam os comportamentos que os produziram $v s$. aqueles que não afetam; produtos que afetam sem mediação vs. aqueles que afetam com mediação os comportamentos que os produziram; e produtos agregados gerados por contingências entrelaçadas $v s$. aqueles gerados por contingências não-entrelaçadas. Essas distinções podem ser sobrepostas resultando em cinco diferentes tipos de produções agregadas, representadas na Figura 2.

Em casos simples, como o da locomoção da pedra de uma tonelada, envolvendo poucos indivíduos se comportando de maneira entrelaçada e um resultado imediato de grande magnitude, o produto agregado pode afetar os comportamentos que o produziram sem mediação, já que, nesse caso, ele coincide com as consequências comportamentais (primeira linha da Figura 2).

Quando um produto agregado é gerado pelos comportamentos - entrelaçados ou não (o que é mais frequente) - de um

5 Esta distinção separa metacontingências e macrocontingências (Glenn, 2004; Malott \& Glenn, 2006). 
número maior de pessoas e/ou é atrasado, cumulativo, ou de pequena magnitude para os indivíduos, essa coincidência de produto agregado e consequência tende a ser mais rara. Nesses casos, os efeitos do comportamento de um único indivíduo raramente terão um impacto discernível no produto agregado (Glenn, 2004; Malott \& Glenn, 2006). Como uma resposta individual não afeta sensivelmente o produto agregado para o qual contribui, o produto agregado não tem efeito comportamental direto sobre a ação, isto é, não se constitui um evento ambiental para o indivíduo cujo comportamento contribui para o produto agregado. Produtos agregados desse tipo geralmente não afetam os comportamentos que os produziram, continuando a serem produzidos enquanto as contingências individuais se mantiverem. Essa característica assinalaria a importância desse tipo de produção agregada: resultados perigosos de nossas ações que não afetam essas mesmas ações são potencialmente catastróficos (segundo Glenn, 2004, envolvem a "tragédia do bem comum"). Tais produções agregadas são diagramadas na terceira linha da Figura 2.

Produtos agregados, no entanto, podem exercer funções comportamentais para outras classes de respostas e/ou para outras pessoas que não as responsáveis pela geração do produto (segunda linha da Figura 2). Nesse caso, as características da retroação do produto agregado sobre os comportamentos daqueles envolvidos em sua produção dependerão dos comportamentos mediadores envolvidos (sejam de membros do próprio grupo produtor ou não). Analogamente ao comportamento individual, produções agregadas envolvendo retroação podem tanto ser selecionadas pelos seus produtos agregados e recorrer (ou re-ocorrer), como, pelo contrário, podem se enfraquecer e desaparecer.

Outro modo de diferenciar produções agregadas envolve classificar as relações entre os comportamentos que geram o produto agregado (Malott \& Glenn, 2006). A coluna direita da Figura 2 representa pessoas se comportando de modo independente (sem entrelaçamento). Isto é, os eventos que participam das contingências tríplices de uma pessoa não integram as contingências de outras pessoas que também contribuem para o produto agregado; as contingências não são entrelaçadas. Nesses casos, qualquer mudança na produção agregada dependerá de alterações em cada uma das contingências tríplices individuais e os comportamentos dos participantes poderão ser inclusive não sociais (ao menos teoricamente).

Sempre que o produto agregado coincidir com consequências comportamentais (primeira linha da Figura 2), contudo, haverá entrelaçamento entre as contingências tríplices envolvidas, já que pelo menos a consequência de todas as contingências (e.g., a locomoção da pedra) será produzida também pelas ações de outras pessoas. Além disso, os participantes da produção agregada podem interagir, isto é, as contingências podem ser entrelaçadas quando os produtos agregados não retroagem ou quando retroagem sobre os comportamentos que os produzem apenas com mediação (segunda e terceira linhas da coluna da esquerda da Figura 2). Em qualquer hipótese, o entrelaçamento complexifica as relações envolvidas na produção agregada, já que uma alteração em uma das contingências tríplices pode afetar tanto o produto agregado como outras contingências envolvidas na produção. A estrutura do entrelaçamento determinará a maior ou menor facilidade de alterar a produção agregada.
Ao se definirem pelo resultado das ações de mais de uma pessoa, as produções agregadas envolvem um recorte de análise distinto do utilizado para o comportamento social, sugerem a necessidade de novos conceitos (como o de produto agregado) e englobam eventos com características e complexidades distintas. A classificação aqui proposta sugere que a compreensão e a intervenção sobre diferentes produções agregadas podem demandar diferentes ferramentas analíticas. Em alguns casos, o recurso à contingência tríplice pode ser necessário e suficiente, enquanto em outros, a retroação do produto agregado exigiria uma análise mais ampla que toma como seu objeto as ações e produtos gerados por mais de um indivíduo (envolvendo os conceitos de metacontingência e macrocontingência, por exemplo).

\section{Prática Cultural}

Um terceiro conjunto de eventos nomeado como fenômeno social abrange os objetos de estudo das Ciências Sociais usualmente englobados por termos como "cultura" e "prática cultural", ou o nível cultural de seleção do comportamento por consequências (Skinner, 1981). Duas características parecem despertar um interesse especial por tais eventos. Uma característica é a repetição ou manutenção de ações similares ao longo de "gerações" de participantes. De fato, é fundamentalmente a manutenção de comportamentos similares mesmo com a substituição dos participantes que diferencia as práticas culturais dos demais fenômenos sociais. A outra característica é chamada de transmissão de modos de agir ${ }^{7}$. Se um grupo de pessoas que interage durante algum tempo tem seus participantes substituídos e suas ações permanecem semelhantes aos dos antigos membros, isso pode se dever a duas coisas: os novos membros entraram em contato com circunstâncias semelhantes àquelas as quais os membros anteriores estiveram expostos, ou - o que é mais provável - os membros antigos de algum modo ensinaram aos novos membros como agir. Os fenômenos sociais que envolvem essa segunda situação tendem a atrair maior interesse na discussão da cultura.

Assim, o que parece fundamental na distinção entre os outros fenômenos sociais e as práticas culturais é a propagação de comportamentos aprendidos similares por sucessivos indivíduos. A expressão "propagação" indica que certo indivíduo A afeta um indivíduo $\mathrm{B}$ de modo a produzir em B um comportamento similar ao seu, ou ao de um terceiro indivíduo $\mathrm{C}$. O indivíduo $\mathrm{B}$, por sua vez, posteriormente afeta outros indivíduos de modo a propagar o mesmo comportamento, e assim por diante. Os "comportamentos" referidos na definição são sempre aprendidos, e poderiam

6 Em discussões sobre a evolução biológica das espécies, o termo "geração" é usado em um sentido próximo ao seguinte: "espaço de tempo correspondente ao intervalo que separa cada um dos graus de uma filiação e que é avaliado em cerca de 25 anos" (Houaiss, Villar \& Franco, 2001, p. 1446). Na discussão de práticas culturais, o termo também é ocasionalmente utilizado. Para evitar confusões com o primeiro uso, porém, não utilizamos esse termo ao tratar de práticas culturais.

7 O termo "transmissão" pode implicar ocasionalmente em propagação por meio de seleção filogenética. Como nosso foco não é nesse nível de seleção, evitamos utilizar esse termo ao tratar de práticas culturais. 
ser, em princípio, respondentes condicionados ou envolver componentes respondentes condicionados; no entanto, as práticas culturais de maior interesse parecem sempre envolver comportamento operante.

O termo "similares", por sua vez, ressalta que as ações envolvidas devem participar de uma mesma classe, definida em termos funcionais. Essa similaridade não exclui a possibilidade ressaltada por Glenn (2003):

Podem ocorrer variações nas consequências (assim como variações nos próprios atos) à medida que as contingências comportamentais são replicadas [ou propagadas] em sucessivos repertórios [comportamentais individuais]. De fato, variações em qualquer um ou em todos os elementos das contingências comportamentais replicantes contribuiriam bastante para a variação necessária à rápida evolução algumas vezes vista nas culturas. (p. 232)

A expressão "sucessivos indivíduos", por fim, ressalta a necessidade de haver substituição dos participantes da prática. Os participantes não precisam manter relação de descendência entre si, isto é, não precisam ser de "gerações" distintas no sentido biológico do termo. A propagação da prática, além disso, não precisa ser mantida por um período de tempo especialmente longo. Muitas ações propagadas entre indivíduos de uma mesma faixa etária e mantidas apenas por um curto período de tempo podem ser consideradas propriamente práticas culturais (e.g., uma "moda jovem de verão").

Recorrendo a Glenn (1991, 2003), então, podemos enumerar como características definidoras do fenômeno que denominamos prática cultural:

1) Engloba principalmente comportamentos operantes, sensíveis às suas consequências - mas também pode envolver relações respondentes condicionadas;

2) Envolve comportamentos funcionalmente similares de mais de uma pessoa;

3) Os comportamentos similares propagam-se através de (são emitidos por) sucessivos indivíduos por meio de processos de aprendizagem;

4) Já que a propagação de certos comportamentos necessariamente implica o entrelaçamento de contingências, os comportamentos envolvidos são sociais ou englobam pelo menos componentes sociais.

Essa definição de prática cultural está articulada a uma abordagem dos fenômenos sociais semelhante àquela descrita aqui para comportamento social, porque coloca sob foco de análise os comportamentos dos membros participantes, mas agora em outro patamar, uma vez que, por definição, práticas culturais envolvem comportamentos de vários indivíduos e o processo de propagação desses comportamentos.

As características definidoras de uma prática cultural permitem a inclusão sob esse rótulo de fenômenos de diversos níveis de complexidade. De fato, "comportamento não social", comportamento social e produções agregadas podem ser propagados através de sucessivos indivíduos e constituírem-se, portanto, em práticas culturais. A complexidade de uma prática cultural seria determinada, assim, pelo menos em parte, pelos fenômenos comportamentais que a compõem.
Um exemplo "simples", muitas vezes citado, e que apresenta todas essas características pode servir para discutir esses diferentes níveis de complexidade. Pesquisadores japoneses observaram durante anos um grupo de macacos (Macaca fuscata) em uma ilha (Itani, Miyadi, Itani \& Nishimura, conforme citados por Harris, 1980; Chauvin \& Berman, 2004). Os pesquisadores atraíam os macacos para a costa com batatas-doces para facilitar a observação. Em certo momento, uma jovem macaca lavou as batatas-doce na água antes de comê-las, ao invés de limpá-las no próprio pelo, e, então, passou a fazê-lo sempre. Após nove anos, entre $80 \%$ e $90 \%$ dos macacos do grupo estavam lavando as batatasdoce. Pela nossa definição, esse seria um exemplo de seleção de uma prática cultural. Três características, entretanto, nos permitem descrevê-lo como uma prática cultural "simples": (1) o comportamento propagado era emitido individualmente já que só entrava em contato com o ambiente físico; (2) a prática não gerava um produto agregado; e (3) a propagação da prática provavelmente envolveu um modo elementar de imitação - um processo comportamental simples quando comparado, por exemplo, com a instrução verbal.

Cada uma dessas características, por sua vez, permite vislumbrar complexidades que outras práticas culturais podem apresentar. A primeira delas é que muitas vezes o que é propagado como uma prática cultural não é um único comportamento que pode ser realizado individualmente (como o lavar batatas-doce), mas um conjunto entrelaçado de comportamentos de mais de um indivíduo. Nesses casos, o que é propagado são sequências ou combinações específicas de comportamentos sociais de diferentes pessoas e não só uma classe de respostas singular e independente. Tais conjuntos de contingências entrelaçadas podem envolver tanto efeitos relevantes sobre o ambiente físico quanto sobre outras pessoas do grupo. Em segundo lugar, práticas culturais também se tornam mais complexas quando geram um produto agregado. Por fim, a propagação de práticas culturais não se restringe à imitação; outros processos mais complexos podem estar envolvidos; no caso humano, por exemplo, comportamento verbal (especialmente instruções) parece estar quase sempre envolvido.

Essa caracterização do que seriam práticas culturais é bastante ampla, englobando boa parte das ações humanas. Dado que quase todo nosso repertório é construído em ambientes sociais culturalmente diferenciados, prever, interpretar e interferir sobre práticas culturais é um desafio teórico e prático da maior relevância. A consecução desses objetivos, entretanto, defronta-se com uma questão ainda não resolvida: como lidar com a complexidade característica das culturas.

\section{A complexidade das culturas: diálogos com as Ciências Sociais}

As práticas culturais mais relevantes atualmente são extremamente complexas e encontram-se fortemente relacionadas a diversas outras práticas que compõem nossas sociedades. Apesar dos mecanismos básicos envolvidos na sua seleção poderem funcionar exatamente da mesma forma que com práticas mais simples, a análise e intervenção sobre tais práticas requer maneiras de lidar com sua complexidade e seu 
enredamento com diversas outras práticas. Isto é, conceitos voltados para o manejo de conjuntos complexos de práticas culturais inter-relacionadas (culturas) podem ser necessários para que perguntas relevantes possam ser respondidas: Terão todas as práticas de uma cultura a mesma importância para a sobrevivência da sociedade? Como as diversas práticas de uma cultura se relacionam? Como uma cultura surge e muda, ou seja, como suas práticas são selecionadas e evoluem? A evolução cultural seria guiada mais fortemente por algum conjunto de práticas culturais?

A discussão sobre a complexidade e a evolução das culturas tem uma longa história nas Ciências Sociais, com a investigação das práticas culturais características de povos específicos e o acúmulo de grande quantidade de dados sobre sociedades complexas - o que pode ser uma grande vantagem ao abordar essa questão. Apesar da grande base empírica, contudo, diversas abordagens teóricas incompatíveis convivem nas Ciências Sociais. Algumas se aproximam mais dos pressupostos da Análise do Comportamento do que outras. Para discutir a questão da complexidade e da evolução das culturas, uma abordagem antropológica em particular tem sido apontada como profícua: o Materialismo Cultural do antropólogo Marvin Harris (Catania, 1984; Glenn, 1988; Guerin, 1992; Harris, 2007; Lloyd, 1985; Malagodi, 1986; Malott, 1988; Vargas, 1985).

Dois aspectos da obra de Harris são especialmente relevantes para as presentes questões: sua ênfase na análise de conjuntos integrados de práticas culturais e seu princípio do determinismo infraestrutural. $\mathrm{O}$ primeiro aspecto reafirma a relevância da obra deste autor para a discussão sobre a complexidade das culturas. Quanto ao princípio do determinismo infraestrutural, é necessário descrever brevemente outra faceta de sua obra antes de apresentá-lo.

As propostas de Harris (1979) para descrever a cultura e a evolução cultural partem de uma distinção do que seriam "dois tipos radicalmente diferentes de fenômenos" (p. 31): eventos "mentais" e eventos "comportamentais". Harris ainda distingue duas perspectivas metodológicas, a partir das quais os eventos que são importantes de serem estudados quando se toma a cultura como objeto de estudo poderiam ser abordados: a perspectiva dos próprios participantes (emic) e a de outros observadores, por exemplo, de antropólogos $(\text { etic })^{8}$.

É a partir dessas distinções, mas enfatizando a relevância dos eventos "comportamentais" e da perspectiva de observadores externos, que Harris (1979) discute a evolução cultural e o princípio do determinismo infraestrutural. Tal princípio envolve a suposição de que as práticas culturais de

8 Glenn (1988), ao discutir essa classificação, apontou que ela não se adequa à perspectiva analítico-comportamental e propôs uma "tradução" dos termos apresentados por Harris. Para essa autora, os fenômenos "comportamentais" descritos por Harris envolveriam comportamento aberto não-verbal, enquanto os fenômenos "mentais" englobariam comportamento verbal (aberto ou encoberto) e outros comportamentos encobertos. A perspectiva etic (de outros observadores) de análise, por sua vez, envolveria a busca pelo estabelecimento de regras passíveis de validação empírica independentemente da comunidade verbal em que surgiram, enquanto a perspectiva emic (dos próprios participantes) envolveria a busca pelo estabelecimento de regras propagadas dentro de uma comunidade verbal particular e que podem ou não ser empiricamente testáveis. um grupo ou, melhor, de uma cultura/sociedade ${ }^{9}$ podem ser divididas significativamente em três grandes "componentes ou setores" que configuram a estrutura universal das culturas: infraestrutura, estrutura e superestrutura (Harris, 1979). Essa estrutura tripartite das culturas se basearia "nas constantes biológicas e psicológicas da natureza humana e na distinção entre pensamento e comportamento e entre as perspectivas emic e etic" (Harris, 1979, p. 51).

Para Harris (1979), todas as sociedades precisam garantir seus requisitos mínimos de sobrevivência por meio de certos modos de produção: tecnologias e práticas empregadas na produção de comida e de outras formas de energia. Todas as sociedades também buscam evitar aumentos ou reduções destrutivas no tamanho de sua população, por meio de modos de reprodução: tecnologias e práticas empregadas para esse fim, como práticas de casamento, cuidado de crianças, contracepção e aborto. Os modos de produção e de reprodução de cada sociedade formam a infraestrutura da cultura.

Todas as sociedades, além disso, precisam manter relações ordenadas entre seus grupos constituintes e com outras sociedades (sobretudo em termos da distribuição do trabalho e dos produtos do trabalho entre indivíduos e grupos) por meio de economias domésticas e políticas: por exemplo, estrutura familiar e de classe, divisão do trabalho, papéis de gênero e educação doméstica e política. Esses conjuntos de práticas culturais constituem a estrutura da cultura.

Por fim, Harris (1979) argumenta que em todas as sociedades há atividades estéticas, esportivas, religiosas e intelectuais. Todas essas atividades e mais quaisquer perspectivas emic e fenômenos "mentais" (de acordo com a classificação de Harris), mesmo aqueles associados à infraestrutura e à estrutura, constituem a superestrutura da cultura (incluindo práticas tais como: noções do senso comum, estruturas de parentesco, ideologias, símbolos, mitos, padrões estéticos, religiões e tabus).

O princípio do determinismo infraestrutural descreveria as relações entre esses três componentes das culturas. Segundo esse princípio, a infraestrutura determina probabilisticamente a estrutura que, por sua vez, determina probabilisticamente a superestrutura. $\mathrm{O}$ determinismo infraestrutural é formulado como uma afirmação da prioridade das relações a serem investigadas e não como um pressuposto irrefutável:

A infraestrutura, em outras palavras, é a principal interface entre cultura e natureza, a fronteira através da qual as limitações ecológicas, químicas e físicas às quais a ação humana está submetida interagem com as principais práticas socioculturais que almejam superar ou modificar aquelas limitações. A ordem das prioridades materialistas culturais da infraestrutura para os outros componentes comportamentais e finalmente para a superestrutura mental reflete a distância cada vez maior desses componentes da interface cultura/natureza. Como o objetivo do materialismo cultural, de acordo com a orientação da ciência no geral, é a descoberta da maior quantidade possivel

9 Harris (1980) define sociedade como "um grupo de pessoas que compartilham um habitat comum e dependem umas das outras para sua sobrevivência e bem estar" (p. 106) e define cultura como "um estilo de vida socialmente adquirido de um grupo de pessoas que inclui modos padronizados, repetitivos, de pensar, sentir e agir" (p. 106). Neste trabalho tomaremos tais termos como equivalentes. 
de ordem no seu campo de investigação, a prioridade para a construção teórica assenta-se logicamente naqueles setores sob as maiores restrições diretas dos dados [givens] da natureza. (Harris, 1979, p. 57)

Nessa perspectiva, uma prática estrutural como o nomadismo dos esquimós, por exemplo, deveria ser analisada remetendo-a, em primeiro lugar, a suas relações com práticas infraestruturais como a subsistência baseada em uma fauna e flora selvagens dispersas.

Apesar de vários analistas do comportamento afirmarem aproximações entre o Materialismo Cultural e o Behaviorismo Radical e do princípio do determinismo infraestrutural sugerir problemas de pesquisa interessantes, o diálogo entre esses dois campos não se consolidou e infelizmente ainda não gerou um programa de pesquisa ${ }^{10}$. O que a obra de Harris (e de outros cientistas sociais como Diamond, 1997/2001, 2005) destaca, contudo, é a necessidade de uma análise de práticas culturais e da cultura tratar também das relações entre conjuntos de práticas culturais. Ignorar esse nível de análise pode comprometer qualquer compreensão ou intervenção voltada para comportamentos propagados entre membros de um grupo.

\section{O Estudo dos Três Fenômenos Sociais}

A distinção aqui sugerida entre comportamento social, produção agregada e prática cultural deveria promover uma avaliação mais precisa e sistemática de como os fenômenos sociais vêm sendo pesquisados na tradição da Análise do Comportamento e estimular sua investigação.

A pesquisa, tanto básica quanto aplicada, sobre o comportamento social e alguns tipos de produções agregadas já tem tradição na Análise do Comportamento (Guerin, 1994; Schmitt, 1998). Os estudos sobre práticas culturais, porém, ainda são escassos e não integrados. Isso talvez ocorra porque ainda faltam distinções conceituais como aquelas que tentamos propor ou que autores como Glenn também vêm propondo. Como destacamos, ademais, as propostas de Harris e de outros cientistas sociais também poderiam ser exploradas no sentido de delas se derivar programas de pesquisa sobre a seleção e evolução das culturas e de práticas culturais.

Nesse sentido, a viabilidade e a utilidade da experimentação em laboratório não devem ser menosprezadas (Baum, Richerson, Efferson \& Paciotti, 2004; Martone, 2008; Pereira, 2008; Vichi, 2004, 2005). A experimentação em laboratório é uma alternativa enriquecedora para uma área na qual muitos trabalhos envolvem basicamente a interpretação a respeito de práticas culturais específicas ou teorizações sem embasamento empírico sistemático (vide a discussão de Otero, 2002, sobre a produção do periódico Behavior and Social Issues).

Alguns analistas do comportamento, porém, têm ressaltado a possibilidade das práticas culturais também serem abordadas empiricamente com outros métodos, entre eles os chamados métodos quase-experimentais, comparativos ou

10 Recentemente, entretanto, Ward (2006) conduziu um experimento de laboratório buscando analisar o princípio do determinismo infraestrutural de Marvin Harris. experimentos naturais (Andery, Micheletto \& Sério, 2005; Kunkel \& Lamal, 1991; Lamal, 1991; Mattaini, 1996; Pierce, 1991 $)^{11}$. Em um momento no qual ainda sabemos muito pouco sobre tais fenômenos, e diante do difícil desafio de produzir suas complexas características em laboratório, a exploração de métodos alternativos pode ser especialmente enriquecedora - inclusive como meio de levantar questões a serem analisadas experimentalmente. Esses métodos também merecem ser discutidos e suas vantagens e limitações apreciadas adequadamente.

\section{Referências}

Andery, M. A. P. A. (1990). Uma tentativa de (re)construção do mundo: a ciência do comportamento como ferramenta de intervenção. Tese de Doutorado, Pontifícia Universidade Católica de São Paulo, São Paulo.

Andery, M. A. P. A., Micheletto, N., \& Sério, T. M. de A. P. (2005). A análise de fenômenos sociais: esboçando uma proposta para a identificação de contingências entrelaçadas e metacontingências. Revista Brasileira de Análise do Comportamento, 1, 149-165.

Andery, M. A. P. A., \& Sério, T. M. de A. P. (2006). Comportamento social. Em H. J. Guilhardi \& N. C. de Aguirre (Orgs.), Sobre comportamento e cognição - Vol. 18 (pp. 124-132). Santo André: ESETEC.

Baum, W. M., Richerson, P. J., Efferson, C. M., \& Paciotti, B. M. (2004). Cultural evolution in laboratory microsocieties including traditions of rule giving and rule following. Evolution and Human Behavior, 25, 305-326.

Catania, A. C. (1984). Editorial: Conceivable book reviews. Journal of the Experimental Analysis of Behavior, 42, 165-169.

Chauvin, C., \& Berman, C. M. (2004). Intergenerational transmission of behavior. Em B. Thierry, M. Singh \& W. Kaumanns (Eds.), Macaque societies: A model for the study of social organization (pp. 209-230). Cambridge: Cambridge University Press.

Diamond, J. M. (2001). Armas, germes e aço: os destinos das sociedades humanas (S. S. Costa, C. Cortes, P. Soares, Trads.). Rio de Janeiro: Record. (Trabalho original publicado em 1997)

Diamond, J. M. (2005). Colapso: como as sociedades escolhem o fracasso ou o sucesso (A. Raposo, Trad.). Rio de Janeiro: Record. (Trabalho original publicado em 2005)

Glenn, S. S. (1986). Metacontingencies in Walden Two. Behavior Analysis and Social Action, 5, 2-8.

Glenn, S. S. (1988). Contingencies and metacontingencies: Toward a synthesis of behavior analysis and cultural materialism. The Behavior Analyst, 11, 161-179.

Glenn, S. S. (1991). Contingencies and metacontingencies: Relations among behavioral, cultural, and biological evolution. Em P. A. Lamal (Ed.), Behavior analysis of societies and cultural practices (pp. 39-73). New York: Hemisphere.

Glenn, S. S. (2003). Operant contingencies and the origins of cultures. Em K. A. Lattal \& P. N. Chase (Eds.), Behavior theory and philosophy (pp. 223-242). New York: Klewer Academic/ Plenum.

11 A experimentação natural pode ser tratada como um tipo de delineamento de pesquisa. Consideramos mais adequado, entretanto, tratá-la como um método distinto. 
Glenn, S. S. (2004). Individual behavior, culture, and social change. The Behavior Analyst, 27, 133-151.

Glenn, S. S., \& Malott, M. E. (2004). Complexity and selection: Implications for organizational change. Behavior and Social Issues, 13, 89-106.

Guerin, B. (1992). Social behavior as discriminative stimulus and consequence in Social Anthropology. The Behavior Analyst, $15,31-41$.

Guerin, B. (1994). Analyzing social behavior: Behavior Analysis and the Social Sciences. Reno: Context Press.

Guerin, B. (2001). Individuals as social relationships: 18 ways that acting alone can be thought of as social behavior. Review of General Psychology, 5, 406-428.

Harris, M. (1979). Cultural materialism: The struggle for a science of culture. New York: Vintage.

Harris, M. (1980). Culture, people, nature: An introduction to general anthropology ( $3^{\text {rd }}$ ed.). New York: Harper \& Row.

Harris, M. (2007). Cultural materialism and behavior analysis: Common problems and radical solutions. The Behavior Analyst, 30, 37-47.

Houaiss, A., Villar, M. de S., \& Franco, F. M. de M. (2001). Dicionário Houaiss da língua portuguesa. Rio de Janeiro: Objetiva.

Keller, F. S., \& Schoenfeld, W. N. (1950). Principles of Psychology. New York: Appleton-Century-Crofts.

Kunkel, J. H., \& Lamal, P. A. (1991). The road ahead. Em P. A. Lamal (Ed.), Behavioral analysis of societies and cultural practices (pp. 243-247). New York: Hemisphere.

Lamal, P. A. (1991). Behavioral analysis of societies and cultural practices. Em P. A. Lamal (Ed.), Behavioral analysis of societies and cultural practices (pp. 3-12). New York: Hemisphere.

Lloyd, K. E. (1985). Behavioral anthropology: A review of Marvin Harris' "Cultural Materialism". Journal of the Experimental Analysis of Behavior, 43, 279-287.

Malagodi, E. F. (1986). On radicalizing behaviorism: A call for cultural analysis. The Behavior Analyst, 9, 1-17.

Malott, M. E., \& Glenn, S. S. (2006). Targets of intervention in cultural and behavioral change. Behavior and Social Issues, 15, 31-56.

Malott, R. W. (1988). Rule-governed behavior and behavioral anthropology. The Behavior Analyst, 11, 181-203.

Martone, R. C. (2008). Efeitos de consequências externas e de mudanças na constituição do grupo sobre a distribuição dos ganhos em uma metacontingência experimental. Tese de Doutorado, Universidade de Brasília, Brasília.

Mattaini, M. A. (1996). Public issues, human behavior, and cultural design. Em M. A. Mattaini \& B. A. Thyer (Eds.), Finding solutions to social problems: Behavioral strategies for change (pp. 13-40). Washington, D.C.: APA.
Otero, M. R. (2002). O compromisso do analista do comportamento com as questões sociais: uma análise a partir de publicações. Dissertação de Mestrado, Pontifícia Universidade Católica de São Paulo, São Paulo.

Pereira, J. M. C. (2008). Investigação experimental de metacontingências: separação do produto agregado e da consequência individual. Dissertação de Mestrado, Pontifícia Universidade Católica de São Paulo, São Paulo.

Pierce, W. D. (1991). Culture and society: The role of behavioral analysis. Em P. A. Lamal (Ed.), Behavioral analysis of societies and cultural practices (pp. 13-37). New York: Hemisphere.

Schmitt, D. R. (1998). Social behavior. Em K. A. Lattal \& M. Perone (Eds.), Handbook of research methods in human operant behavior (pp. 471-505). New York: Plenum Press.

Skinner, B. F. (1953). Science and human behavior. New York: Free Press.

Skinner, B. F. (1978). Walden II: uma sociedade do futuro (2a ed.) (R. Moreno \& N. R. Saraiva, Trads.). São Paulo: EPU. (Trabalho original publicado em 1948)

Skinner, B. F. (1981). Selection by consequences. Science, 213, 501-504.

Skinner, B. F. (1992). Verbal behavior. Acton: Copley. (Trabalho original publicado em 1957)

Vargas, E. A. (1985). Cultural contingencies: A review of Marvin Harris' "Cannibals and Kings". Journal of the Experimental Analysis of Behavior, 43, 419-428.

Vichi, C. (2004). Igualdade ou desigualdade em pequeno grupo: um análogo experimental de manipulação de uma prática cultural. Dissertação de Mestrado, Pontifícia Universidade Católica de São Paulo, São Paulo.

Vichi, C. (2005). Igualdade ou desigualdade: manipulando um análogo experimental de prática cultural em laboratório. Em J. C. Todorov, R. C. Martone \& M. B. Moreira (Orgs.), Metacontingências: comportamento, cultura e sociedade (pp. 86100). Santo André: ESETec.

Ward, T. A. (2006). An experimental analysis of Harris's Cultural Materialism: The effects of various modes of production on metacontingencies. Dissertação de Mestrado, Stephen F. Austin State University, Nacogdoches, Texas.

Recebido em 29.10.08

Primeira decisão editorial em 17.03.09

Versão final em 16.04.09

Aceito em 03.06.09 